\title{
La retórica de las matemáticas en los tratados aritmético-algebraicos del Renacimiento
}

\section{The rhetoric of mathematics in arithmetic and algebraic books of the Renaissance}

ITZIAR MOLINA SANGÜESA

Departamento de Lengua Española

Facultad de Filología

Universidad de Salamanca

Plaza de Anaya s/n. Salamanca, 37008

itziarmolina@usal.es

Orcid ID 0000-0002-5520-3783

Resumen: El profundo movimiento de transformación y renovación de los órdenes social, económico y cultural acaecido a finales del Medievo, así como el dinamismo económico de los siglos XV y XVI y el afán de riqueza de la clase burguesa propició, además de una rápida expansión urbana y un incremento de las relaciones comerciales continentales, la imperiosa necesidad de aprender aritmética para poder llevar a cabo con éxito la nueva contabilidad. En consecuencia, con el fin de satisfacer estas necesidades sociales y en aras de llegar al mayor público posible, durante el Renacimiento se publicaron, por vez primera en español, multitud de obras consagradas a las cuentas. La redacción de estos tratados aritmético-algebraicos -cuya finalidad era eminentemente práctica- en la centuria quinientista trajo consigo no solo la democratización del cálculo, sino que, además, desde un punto de vista lingüístico, dignificó el empleo de las lenguas vulgares como transmisoras de saberes tradicionalmente expresa- dos en latín a una minoritaria élite culta y favoreció la construcción de un tipo de discurso cuya retórica pretendemos analizar en este trabajo. En suma, nuestro objetivo consiste en poner de manifiesto, mediante fragmentos extraídos de los tratados más significativos del siglo XVI que contienen aritmética y álgebra, las características estilísticas y divulgativas de este registro léxico especializado. Motivados por la corriente pedagógica humanista y por el fin de aleccionar (docere), destacan en el corpus estudiado el uso de multitud de ejemplos, citas y alusiones a autoridades, instrucciones y advertencias, entre otros recursos retóricos que consideramos claves tanto para el asentamiento y difusión de estas disciplinas en la Península Ibérica como para la configuración del tecnolecto matemático en lengua española.

Palabras clave: Lenguaje científico. Retórica. Matemáticas. Renacimiento. 
Abstract: The deep movement of transformation and renewal of the social, economic and cultural orders befallen in the late Middle Ages, as well as the economic dynamism of the fifteenth and sixteenth centuries and the eagerness of wealth from of the bourgeois class contributes, along with a rapid urban expansion and an increase of continental commercial relationships, the urgent need to learn arithmetic in order to carry out successfully the new accounting. Consequently, in order to satisfy those social needs and for the sake of arrive to the greater number of readers, during the Renaissance were published, first in Spanish, many works devoted to the calculations. The publication of these arithmetic-algebraic treatises - whose purpose was essentially practical- in the five-hundred century brought with it, not only the democratization of the calculation, but also, from a linguistic viewpoint, dignified the use of the vernacular lan- guages as transmitters of knowledge expressed traditionally in Latin to a minority elite and favored the construction of a type of speech whose rhetoric we analyze in this paper. In short, our aim is bring to light, through extracted fragments of the most significant sixteenth century treatises that containing arithmetic and algebra, the stylistic and informative characteristics of this specialised vocabulary. Motivated by humanist pedagogical theory and to instruct (docere), highlighted in the corpus studied the use of many examples, citations and allusions to authorities, instructions and warnings, among other rhetorical resources which we consider key to the establishment and dissemination of these disciplines in the Iberian Peninsula just like the configuration of the mathematical technolect in Spanish.

Keywords: Scientific Language. Rethoric. Mathematics. Renaissance.

\section{INTRODUCCIÓN}

\subsection{La relevancia de las ciencias exactas en el Renacimiento}

a profunda transformación y renovación de los órdenes social, económico y cultural acaecida a finales del Medievo culminará en el despegue científicas en Europa. Sin embargo, como apunta Maravall (68), esta nueva realidad del estado moderno no hubiera sido posible sin el "espíritu de cálculo" que, extendido a todos los ámbitos socio-culturales, llegó a producir un "auténtico proceso de aritmetización de la realidad" y una configuración del saber alejada del trivium y quadrivium medievales.

Por su parte, el dinamismo económico de los siglos XV y XVI y el afán de riqueza de la clase burguesa desencadenaron una auténtica revolución comercial (origen del moderno capitalismo) que propició una rápida expansión urbana y un incremento de las relaciones comerciales continentales. En este contexto nace la imperiosa necesidad de aprender aritmética; concretamente, cálculo mercantil, instrumento ineludible para llevar a cabo con éxito la nueva contabilidad. Así, durante el Quinientos, se imprimieron en español multitud de obras dedicadas a las cuentas, cuyo propósito principal era la instrucción del mercader y el uso de la cultura matemática como vía de ascenso y cambio social. 
Desde un punto de vista lingüístico, el empleo del vernáculo castellano por vez primera como vehículo de divulgación de estos contenidos en competencia con la lengua latina trajo consigo un hecho revolucionario: la democratización del cálculo.

En esta línea, el objetivo de este trabajo es poner de manifiesto, mediante fragmentos extraídos -a modo de ejemplo- de los tratados más significativos del siglo XVI que contienen aritmética y algunas nociones de álgebra, las características estilísticas y divulgativas de este registro léxico especializado; aspectos claves tanto para el asentamiento y difusión de estas disciplinas en la Península Ibérica como para la configuración del tecnolecto matemático en lengua española.

\subsection{Los matemáticos hispanos y sus tratados}

$\mathrm{El}$ análisis que presentamos se basa en cinco obras de índole aritmético-algebraica (pertenecientes al corpus del Diccionario de la Ciencia y de la Técnica del Renacimiento, DICTER $)^{1}$ redactadas por los matemáticos hispanos más representativos de la centuria quinientista:

1.2.1 Juan de Ortega. Palentino de origen (Palencia, ca. 1480-¿? 1542), fue miembro de la Orden de Predicadores adscrito a la provincia de Aragón. Enseñó aritmética y geometría en España e Italia, privada y públicamente (Rey 67) y fue el autor de la más importante de las aritméticas mercantiles publicadas en la Península Ibérica: Conpusición de la arte de la Arismética y de Geometría. El propósito de esta obra era esencialmente de carácter práctico y didáctico, para que "no passasen tantos fraudes como pasan por el mundo de las cuentas" (fol. 1v), esto es, con la idea "de facilitar la exactitud, tan ligada a la honestidad en las prácticas mercantiles, conforme a las virtudes que en su profesión los burgueses deben cultivar" (Maravall 167).

1.2.2 Marco Aurel. Entre los pocos datos que se conocen sobre su biografía destaca su origen alemán. Afincado en Valencia, ejerció, según detalla en sus obras, la profesión de maestro de contar. En el ecuador del siglo que nos ocupa, publicó la primera obra impresa en España cuyos contenidos versan

1. Formado por 74 obras científico-técnicas, en cuya selección se contó con el asesoramiento de especialistas en la historia o arqueología de las diferentes disciplinas. Actualmente digitalizado $\mathrm{y}$ accesible en $<\mathrm{http}: / /$ dicter.usal.es $>$. 
sobre la regla de la cosa o álgebra. ${ }^{2}$ De hecho, certifica en el prólogo el carácter innovador de su obra al admitir que "es cosa nueva lo que trato y jamás vista ni declarada, y podrá ser que ni aun entendida ni imprimida en España" (fol. IIIr); de ahí que se le haya considerado tradicionalmente como el introductor del álgebra en el marco hispánico del Renacimiento. Este tratado, titulado Libro primero de Arithmética algebrática, ejerció una gran influencia en el desarrollo de esta vertiente abstracta de las matemáticas en la Península Ibérica, ya que el resto de textos con contenidos algebraicos publicados en este territorio a lo largo del siglo XVI se basaron en el compuesto por el germano, como puede apreciarse en la Arithmética de Antic Roca (1565) o en la de Juan Pérez de Moya (1562). ${ }^{3}$

1.2.3 Juan Pérez de Moya. Matemático andaluz (Santisteban del Puerto, Jaén, ca. 1513-Granada, 1597), se formó en Alcalá de Henares y Salamanca, en cuya ciudad, detalla el maestro Alejo de Venegas, "con público applauso ha leýdo", al igual que lo hizo "en la Corte y en otros muchos lugares insignes". ${ }^{4}$ Pérez de Moya fue, como aseguran sus biógrafos, un hombre extraordinariamente culto que leyó y asimiló todo lo publicado en su época. Asimismo, fue un autor prolífico y variado que publicó multitud de textos, tanto de temática científico-matemática como religioso-moral (Rodríguez Vidal 7-13). Ahora bien, tal y como certifica la valoración de la crítica especializada, ${ }^{5}$ fue en su faceta de matemático en la que más destacó.

Su obra más relevante, Arithmética práctica y speculativa, fue publicada por primera vez en 1562 y llegó a alcanzar más de 30 ediciones hasta 1875. Este tratado, que contiene, a lo largo de nueve libros, aspectos de aritmética teórica y práctica, es considerado por los estudiosos como uno de los libros "más importantes en la España del siglo XVI, no tanto por sus innovaciones (que no las tiene) sino por lo que supuso en la divulgación de esta materia, tenida por muchos como excesivamente árida y, por ende, inaccesible" (Valladares 391).

2. Aunque no la primera que se escribía (ver Docampo).

3. La intertextualidad existente entre la obra de Aurel y la de Pérez Moya ha sido tratada en Molina (2015a).

4. Ver prólogo de la Arithmética práctica y speculativa (XIII-XV).

5. Por ejemplo, Picatoste (245) afirma que "Moya fue un matemático distinguido y profundo que reunió en sus obras, con gran criterio, cuanto entonces se sabía de estas ciencias, aclarando muchos conceptos y buscando demostraciones ingeniosas y resoluciones breves y sencillas a los problemas de mayor aplicación [...]. Formó parte un grupo de hombres eminentes que luchó tenazmente en España, durante todo el siglo XVI, por vencer el odio, el desprecio o el temor al estudio de las ciencias". 
Asimismo, otro de los textos matemáticos de Moya digno de ser destacado es el manual de aritmética práctica que confeccionó destinado a aquellos que aspiraban a desempeñar el oficio de contadores o computistas,

en que se pone, en suma, lo que un contador ha menester saber, y una orden para que los que no saben escrivir, con oyrlo leer, sepan contar y convertir de memoria unas monedas en otras. Con unas tablas al fin en guarismo y castellano, para averiguar con facilidad las cuentas de los réditos de los censos y juros, según usança de España y otros Reynos. (1589 portada)

Este tratado sigue el modelo de los manuales de mercadería surgidos en el seno de ciertas empresas mercantiles europeas, en cuyas páginas los valores, así como las paridades monetarias y los tipos de cambio encuentran un lugar destacado, auspiciados probablemente por "el afán de cálculo y de exactitud que empezó a inundar la mentalidad mercantil y urbana de la época" (Salavert 87).

1.2.4 Pedro Núñez Salaciense. Afamado cosmógrafo y matemático portugués (Alcácer do Sal, 1502-Coimbra, 1578), estudió Filosofía y Medicina en Lisboa, donde obtuvo el grado de Doctor y explicó una cátedra de Filosofía desde 1530 hasta 1533. Asimismo, se aplicó con ahínco al estudio y cultivo de las ciencias exactas, motivo por el que obtuvo la primera cátedra de Coimbra en 1544 (Picatoste 218) y fue durante seis años profesor de Matemáticas en la Universidad de Salamanca (Flórez 418). Fue, sin duda, como afirma Leitão (9), "um dos matemáticos europeus de maior fama no século XVI"; fama muy merecida, ya que "enriqueció la matemática con varias ideas verdaderamente geniales, que lo colocan a una altura inmensa sobre los demás matemáticos españoles y portugueses de aquella época, y quizás de todos los tiempos" (Rey 115). Por ejemplo, entre las obras de Aurel o Moya y el Libro de Álgebra en Arithmética y Geometría de Núñez existe una profunda diferencia: en estas dos primeras el álgebra es un capítulo o apéndice más de la aritmética, casi reducida a la regla de la cosa, aplicada a las diversas igualaciones simples y compuestas, expuestas dogmáticamente. Por el contrario, la obra del portugués, publicada 30 años antes en su lengua materna, según certifica el autor en el prólogo (IIIr-IIIv), supone un gran avance, ya que al dedicar las dos primeras partes al álgebra como tal confiere a este saber una entidad propia, que hasta entonces no se le había concedido. Si tenemos en cuenta, como pone de mani- 
fiesto Flórez (418), que "su libro fue escrito hacia 1537 podemos decir que se anticipó a Cardano, que es considerado como el primero que dio autonomía al álgebra en su obra: Ars Magna, publicada en el año 1545".

\section{ANÁLISIS}

\subsection{Retórica y estilo de los tratados aritmético-algebraicos}

Frente al sistema de enseñanza medieval, cuya instrucción -monopolizada por el clero- tenía como base el esquema de las siete artes liberales -consideradas suma total de la sabiduría secular-, los humanistas, sujetos a la influencia de los ideales retóricos y culturales, provocaron un cambio total en la filosofía;

no solo respecto a las fuentes, sino también en el estilo, en la terminología y en los géneros. Acuñaron para sus estudios (con base en antecedentes clásicos) el término de studia bumanitatis, y formularon ideales educativos y culturales nuevos, que en mucho ayudaron a elevar el prestigio y la influencia de que gozaban. Estos hombres afirmaban haber logrado un renacer de la erudición y las letras, siendo con ello causa del nombre Renacimiento con que hoy conocemos a ese periodo. (Kristeller 162)

En esta época, certifica Kristeller, la retórica evolucionó, creció y llegó a propagarse e invadir todos los campos de saber; especialmente significativa y relevante resultó, como comprobaremos, su función en la transmisión del saber matemático.

El modelo pedagógico humanista, junto con las normas de la retórica clásica -todavía vigentes en la España del siglo XVI (Alburquerque)-, ${ }^{6}$ generó, desde la vertiente de la instrucción técnica aplicada a las ciencias exactas, un tipo de discurso divulgativo eminentemente didáctico. En aras de cumplir con el propósito y objetivo pragmático de sus tratados, los matemáticos de la centuria quinientista se decantaron por uno de los tres fines de la retórica: el docere. De ahí que en el corpus analizado documentemos un tipo de genus demostrativum (Lausberg 212-25), sobre todo, y una profusión de usos de exem-

6. "No es vano el empeño de nuestra retórica del Siglo de Oro en sistematizar de un modo claro lo que ya estaba presente en la retórica greco-latina. Estos memoranda acerca del status «discursivo» recogidos por unos y otros tratados, mantendrán viva la doctrina [...] y serán de vital importancia para el desarrollo posterior de esta ciencia" (57). 
pla o similitudo que avalen los contenidos teóricos, así como una abundante alusión a testimonios de auctoritas (Lausberg 349-59), entre otras características estilísticas de su retórica que en las siguientes páginas reseñamos.

Así pues, los maestros de cuentas y algebristas hispanos del Renacimiento, en numerosas ocasiones, precisan el sentido de las palabras nuevas en sus textos mediante definiciones y glosas explicativas. Asimismo, detallan las instrucciones necesarias para llevar a cabo la aplicación de los contenidos aritmético-algebraicos, seguidas, en algunos casos, de diversas recomendaciones y advertencias, cuyo propósito es fomentar la óptima comprensión de las ciencias exactas; basadas preferentemente en la resolución de problemas, para los cuales aportan una infinidad de demostraciones y ejemplos. De manera análoga, la intertextualidad y la profusión de citas de autoridades son recursos altamente productivos para construir (y dotar de rigor científico) el discurso matemático en la época que nos ocupa. ${ }^{7}$

En suma, características de un discurso científico basado en el principio retórico del aptum o acomodación a las circunstancias, es decir, al dominio o bagaje cultural e intelectual del público al que va dirigido.

\subsubsection{Definiciones}

Los tecnicismos suelen ir encabezados por una definición la primera vez que son usados. La definición es uno de los recursos empleados para facilitar la comprensión de nociones científicas o conceptos abstractos, del mismo modo que las demostraciones y ejemplos de la aplicación de los mismos. De hecho, demostraciones y definiciones son, en palabras de Etayo Miqueo (368), las dos actuaciones en que se desenvuelve la literatura matemática: "la demostración es el relato y la definición, el retrato".

Mediante el empleo de definiciones se intenta, además, la consecución del rigor y precisión que caracteriza a la terminología matemática como sucede, por ejemplo, en las operaciones aritméticas más básicas: ${ }^{8}$

7. Motivados por la finalidad del docere predominante en estos tratados, hemos escogido como objeto de análisis estos seis aspectos. Para más información sobre otros recursos retóricos empleados en la configuración del tecnolecto matemático en lengua española, como las metáforas, ver Molina 2017.

8. El resaltado de las voces en negrita y cursiva, así como los subrayados del ejemplo -y los sucesivos- es nuestro. 
Numerar es saber dezir o esplicar el valor de un qualquier número. Los números, o se escriven con una sola letra de las diez del guarismo, o con muchas. Si se escrive con una sola letra, ya se ha dicho lo que vale cada una. Si se escriviere con muchas, en tal caso cada una letra tendrá valor según el lugar do estuviere. (Pérez de Moya 1589, fols. 7v-8r)

Sumar es juntar muchos números o partidas en una. (Pérez de Moya 1589 , fol. 14v)

El restar es la $3^{\text {a }}$ specie que conviene al Arithmética, y es la $2^{\text {a }}$ de las quatro reglas principales. La qual no es otro sino, de dos números inyguales, buscar la differencia de quánto es mayor o menor el uno que el otro; porque si son yguales, no ay qué hazer. (Aurel fol. 4r)

Multiplicar un número por otro es buscar otro número tercero de tal condición que se aya con el uno de los dos números en la proporción que el otro a la unidad, y al contrario. (Pérez de Moya 1562, 43)

Partir, en quanto al propósito que aquí pretendemos, es dividir o hazer una cantidad o número muchas o pocas partes yguales. (Pérez de Moya 1589 , fol. 61v)

O en la exposición de tipos de números concretos específicos: ${ }^{9}$

Número par es un número que se puede dividir en 2 partes yguales, sin fractión de la unidad, assí como 10, que se divide en dos cincos. Otros lo definen diziendo: número par es que se puede dividir en partes pares y en impares; assí como 10 se divide en 7 y 3 , ó 9 y 1 , ó 6 y 4 ó 8 y 2 . De las quales definiciones carece el número impar. (Pérez de Moya 1562, 321) Número impar es el que no se puede dividir en dos partes yguales sin fractión de la unidad. Otros lo definen diziendo: número impar es que, dividido en qualesquiera partes, la una será par y la otra impar. Assí como 7 se divide en 6 y 1, ó en 4 y 3 , ó en 2 y 5 , a differencia de lo que el primero artículo dize del número par. (Pérez de Moya 1562, 325)

Número racional es un número que tiene raýz discreta, quiero dezir justa, assí como 4, 9, 16, que sus raýzes son 2, 3, 4. (Pérez de Moya 1562, 454) Número superfluo o superante es todo número que es excedido de la summa

9. También se documentan, aunque en menor medida, algunas reformulaciones léxicas del tipo: "Mediano se dize un charácter que está entre dos extremos, uno que le sea mayor y otro menor, assí como co. está entre n. y ce., y el ce. está entre co. y cu., etc., y assí de los semejantes” (Pérez de Moya 1562, 553). 
de sus partes alíquotas; assí como 12, que tiene por partes alíquotas 1 , $2,3,4,6$, la summa de las quales es 16 . Pues porque los 16 sobrepujan al todo (que en este exemplo fue 12), por tanto dirás que el 12 y los que tuvieren su propriedad serán números superantes o superfluos. (Pérez de Moya 1562, 326)

\subsubsection{Glosas explicativas}

Mediante el empleo de glosas explicativas se aclaran e iluminan ciertos conceptos o términos de difícil comprensión, como es el caso de los numerales fraccionarios. De ahí que los matemáticos quinientistas hagan hincapié en su particular modo de expresión, en el que actúan, como es bien sabido, dos números enteros naturales separados por una raya:

Es de saber que para poner qualquiera quebrado en figura se ha de hazer una raya pequeña, d'esta manera: /. (Pérez de Moya 1562, 130)

El numerador nombra diziendo todo lo que está encima de la raya; el denominador denomina el ser de aquello que nombró el numerador, como el exemplo puesto declara, porque el numerador nombra diziendo tres, y el denominador da a entender que los tres que nombró el numerador son quartos. Y esso es lo que quiere dezir el 4 que está debaxo

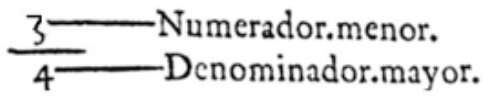

(Pérez de Moya 1562, 131)

Esta figura $1 / 2$ quiere dezir medio, y figúrase assí porque la raya denota tanto como partido, y assí querrá dezir la figura que uno, partido a dos, que están debaxo, cabrá a medio; porque si una cosa se divide en dos partes yguales, qualquiera d'ellas se dirá medio:

\section{Vn medio.}

$$
\frac{\mathrm{I}}{2} \quad \text { (Pérez de Moya 1562, 131) }
$$

De modo similar, explican qué se entiende en sus obras por determinados conceptos (como una raíz cuadrada), de los que detallan, también, cuáles son sus características y naturaleza: 
Por raíz quadrada entendemos un número que, multiplicado por sí mismo, haze otro número, el qual, por essa causa, se llama quadrado, assí como es 2 en respecto de 4, y 3 en respecto de 9, y 4 en respecto de 16 , y 5 en respecto de 25 . Y porque todo número puede ser multiplicado por sí mismo, será, por esta causa, todo número raíz quadrada de otro número, el qual se representa en forma quadrada. Pero no tiene todo número raíz quadrada perfeta y punctual, porque a ninguno d'estos: 2 , $3,5,6,7,8,10,11,12,13,14,15,17,18,19,20$, ny a muchos otros que van procediendo en infinito puede responder algún número que, multiplicado por sí mismo, lo restituya. (Núñez, fol. 43r-v)

El empleo de preguntas sirve, igualmente, a los autores para resaltar e introducir o justificar una explicación que consideran relevante. Además, en determinados contextos se sirven de los paréntesis para precisar o recordar información esencial sobre algunos términos:

Pregunto: ¿estas ocho figuras siguientes, quánto montan? 39541080. Para declaración de lo qual començarás a numerar desde el zero primero que está a la mano derecha, diziendo unidad (que quiere dezir unos), y porque el zero no vale ninguna cosa, dirás que esta primera letra no vale nada. Prosigue adelante, diziendo dezena en la figura siguiente que está después del zero discurriendo hazia la mano izquierda, que es ocho, y porque vale ocho dirás que son ocho diezes, que por otra denominación serán ochenta. Pasa a la tercera figura, que es zero, y di centena (que quiere dezir cientos) y serán tantos cientos quantos la figura a la qual tal nombre dieres valiere unidades, y porque el zero no vale ninguna cosa no avrá ningún ciento [...]. (Pérez de Moya 1562, 10)

¿Para qué se multiplica la partición por lo que se multiplica el partidor? Esto está claro que se haze por acrescentar o disminuyr la partición con la misma quantidad que se acrescentó el partidor. (Pérez de Moya 1562, 542)

\subsubsection{Instrucciones y recomendaciones}

Otra de las características estilísticas esenciales de los tratados matemáticos del Renacimiento hispano es la inclusión de instrucciones en el discurso (normalmente mediante el uso de formas verbales -en imperativo o futuro de indicativo- de la 2. ${ }^{a}$ persona del singular), a través de las cuales indican a un 
'tú' lector o destinatario de la obra cuáles deben ser los pasos que debe seguir para que pueda efectuar correctamente una operación aritmético-algebraica cualquiera. Como puede apreciarse en los siguientes fragmentos, se exponen paso por paso y con detenimiento y minuciosidad las instrucciones propias de cada una de las operaciones matemáticas contenidas en sus textos:

Para multiplicar de binóminos y residuos, pornás un binómino debaxo del otro, o un residuo debaxo del otro, o un binómino debaxo de un residuo, o residuo debaxo de binómino, como quiera que venga. $\mathrm{Y}$ començarás a la mano yzquierda (no porque no puedas començar a la mano derecha, mas a mí me paresce ser más galano. Cada uno haga lo que le paresca). Y multiplica todas las quantidades de arriba con cada una de las de abaxo, $\mathrm{y}$, acabado de multiplicar, summarás cada género con su ygual, como en el summar has visto. (Aurel, fol. 58v)

Si sumando hizieres diez o diezes justos, pocos o muchos, assentarás un solo zero en derecho de lo que fueres sumando, y el diez o diezes guardarlos has para llevarlos otra casa delante, como en la precedente regla se dixo. (Pérez de Moya 1589, fol. 15r)

Pon una suma encima de la otra, como tengo dicho, y haz una raya debaxo de amas sumas y di: 5 vezes 4 son 20; por quanto vienen los diezes yguales, pondrás un zero debaxo de la raya y lleva contigo dos en lugar de de los veynte, para ayuntarle con los dezenales. (Ortega, fol. 15r)

En ciertas ocasiones, los autores del XVI aportan, bien mediante una nota o bien entre comas, algunas recomendaciones que sirven tanto para facilitar el cálculo como para instruir a los usuarios de tratados matemáticos como buenos contadores o algebristas. Estas aparecen expresadas en sentencias del tipo:

Quando summares, procura evitar esta letra $Y$, porque el buen contador no ha de contar con son, quiero dezir que quando fueres summando no digas tanto y tanto es tanto. (Pérez de Moya 1562, 24)

Nota. Para escusar muchas razones en multiplicar alguna quantidad con $\underline{10}$, no harás otro que juntar a la tal quantidad un 0 al cabo de la mano derecha, y verná a estar el dicho 0 en lugar de la unidad, y será ya multiplicada tal quantidad con 10. (Aurel, fol. 6r-v) 


\subsubsection{Avisos y advertencias}

Por otro lado, los textos del corpus manejado se caracterizan por incluir una serie de advertencias o avisos en los que se fundan los autores para prevenir el error del lector/ aprendiz de matemáticas. Por ejemplo, uno de los consejos de Pérez Moya ante la dificultad de la resolución de una operación demandada es recurrir a un modelo análogo preexistente comprendido:

Nota un aviso para quando te dieren alguna qüestión y no entendieras lo que has de hazer. Digo que, a imitación de la mesma demanda que te dieren, ordenes otra con números conoscidos, y en ellos traçarás hasta que saques por regla lo que de memoria sabes que ha de ser, y de la suerte que hizieres la fácil harás la diffícil. (Pérez de Moya 1562, 233-34)

O múltiples avisos del tipo:

Quienquiera que quisiere ayuntar o sumar muchas sumas en uno deve tener este aviso o manera: que todavía quando quisiere sumar o ayuntar muchas sumas en uno, se ponga siempre nonbre enfruente de nonbre, y dezena enfruente de dezena y centena enfruente de centena, y ansí de todas las figuras que se siguieren. (Ortega, fol. 4r)

$\mathrm{Y}$, por tanto, te quiero dar este aviso: que quandoquiera que tú quisieres desminuir dos nombres, conviene a saber, nonbrador y denominador, que por la mesma regla que disminuyres el uno disminuirás el otro, porque en otra manera sería falsa la tal disminuyción, como adelante verás. (Ortega fol. 71r)

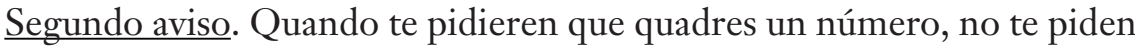
otra cosa sino que le multipliques por sí mesmo. Exemplo. Dame el quadrado de 7. Multiplica 7 por sí mismo, diziendo: 7 vezes 7 hazen 49; este 49 se dize potencia o quadrado del 7. (Pérez de Moya 1562, 469)

Asimismo, testimoniamos, entre otras, las siguientes advertencias:

Advierte lo que has hecho con el 2, porque, si fuere 3, tresdoblarás y añadirás los zeros que vinieren con el tres. Y si fuere 4, quatrodobla; y si 5 , cincodobla, y assí hasta llegar al 9, que nuevedoblarás. (Pérez de Moya 1589 , fol. $155 \mathrm{r}-\mathrm{v}$ ) 
Ahora que para restar qualquiera cuenta, que siempre deve estar en el renglón de arriba la mayor cantidad, agora sea deuda o sea paga, porque en otra manera sería falsa la cuenta. (Ortega, fol. 7r)

Nota. Para que mejor te puedas fiar tal summa ser bien summada, si has començado de summar de arriba hazia baxo, torna a summarla y comiença de abaxo y summa hazia arriba; y si viniere tanto de una manera como de la otra, puedes piadosamente creer tal summa ser bien summada. (Aurel, fol. 2v)

Mas al partir no podrás poner la valor de la co. a tu plazer, hasta tanto que por una ygualación te sea descubierto. (Aurel, fol. 74v)

\subsubsection{Demostraciones y ejemplos}

Inexcusable en el quehacer matemático es la presencia de demostraciones y ejemplos que ilustren y aclaren la aplicación de reglas, pruebas, operaciones, etc. Así, declara Aurel (fol. IIr) que "la sciencia mathemática, entre todas las Artes liberales, [...] es la que con más claras y abundosas demonstraciones se demuestra". Efectivamente, como puede leerse en los fragmentos seleccionados, las demostraciones son la base de las ciencias exactas:

Que la sciencia mathemática, entre todas las Artes liberales, muy Magnífico Señor, sea la más clara y la más cierta y necessaria es cosa evidente, pues sola ella (y principalmente lo que en ella se trata) es la que con más claras y abundosas demonstraciones se demuestra. (Aurel, fol. IIr)

De manera que en los otros libros de Arithmética, assí del autor como agenos, unos mejor que otros enseñan el arte, pero éste enseña por demostración, y evidencia y causas. (Pérez de Moya 1562, 447)

Para demonstración d'esta regla, procederemos continuando la proporción tripla hasta la décima dignidad y aplicaremos nuestra demonstración a un exemplo, como si generalmente demonstrássemos en todos. Porque la regla es general, ora se multiplique una dignidad en sí misma, ora en otra, y la demonstración que sirve en un exemplo sirve en todos, aplicándola como conviene. $\underline{Y}$ parescionos este modo más claro para los que no son acustumbrados a las demonstraciones. 


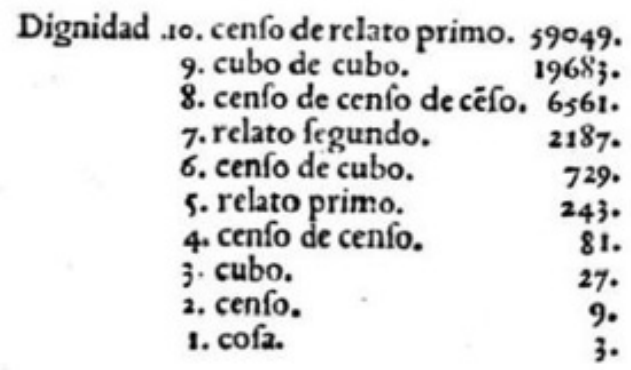

(Núñez, fol. 27r-v)

Por su parte, los ejemplos son concebidos como una herramienta que permite visualizar, comprender y poner en práctica los conceptos que se hallan en el plano abstracto. Por consiguiente, contribuyen a la adquisición del dominio pleno de la disciplina objeto de estudio:

Pues que suficientemente an sido declaradas las tres diferencias que se hallan en el sumar, pues que ya no falta sino saber cómo se sumarán todas tres juntas quando acaheciere, por mayor declaración y bien brevemente, yo pondré un exemplo en el qual se comprehenderán todas tres diferencias [...]. Y ansí, por estos en exemplos sobredichos, podrás sumar qualquiera cuenta grande o pequenya que sea en tal que sea por entero. (Ortega, fol. 5r-v)

Nota que esta regla del partir puede venir en tres maneras, [...]. Y para que mejor lo entiendas, porné de cada una differencia un exemplo. (Aurel, fol. 8r)

Reduzir no es ni quiere dezir otra cosa sino las proporciones abscondidas en los rotos traherlas a perfectión, que es a número entero [...], como por la práctica de los exemplos mejor se entenderá. (Pérez de Moya 1562, 157)

Para hallar el medio arithmético entre dos extremos, junta los dos extremos simplemente y la metad de tal conjuncto será el medio entre tales dos extremos. Exemplo: entre 10 y 2 , ¿quál será el medio? Junta 10 con 2 , serán 12 , cuya metad es 6 . Éste dirás qu'es el medio entre 10 y 2. (Aurel, fol. 18v) 


\subsubsection{Citas de autoridades}

Por último, cabe destacar el papel predominante que ocupan en el discurso matemático las citas o referencias de autoridades -auctoritas tanto históricas como sincrónicas (Lausberg 359)- de este campo del saber. Estas se erigen como la base o los pilares sobre los que se asientan y construyen las ciencias matemáticas. El profuso empleo de citas y la mención explícita de las fuentes originales ponen de manifiesto el impulso a la difusión del saber que caracteriza los textos científico-técnicos del Siglo de Oro español.

A continuación, ponemos de relieve con fragmentos extraídos de estas obras renacentistas dos características principales. Por un lado, la tradición, ${ }^{10}$ reflejada en la mención de autoridades clásicas, entre las que destacan los filósofos pitagóricos, Platón y Aristóteles o los matemáticos greco-latinos Boecio y Euclides, para la difusión de contenidos relacionados con la aritmética y la geometría. Y, por otro lado, la innovación, que emana de la influencia ejercida por las obras de algunos autores italianos coetáneos, fundamentales para la divulgación y desarrollo del álgebra.

\subsubsection{Antiguos}

Como aparece manifiesto en los párrafos siguientes, tanto los sistemas de numeración como algunas técnicas de contabilidad parten de la tradición. Así pues, se alude a "los antiguos" o "antepasados" en general y a diversos filósofos y matemáticos griegos en particular:

El octavo tracta modos de contar que tuvieron los antiguos, y de monedas, y pesos y declaraciones de muchos characteres que se ponían por números, con otras muchas antigüedades, juntamente con el cómputo para sacar las fiestas que dizen movibles. (Pérez de Moya 1562, XI)

Para escrivir quinientos, ponían esta figura: D, por razón que los antiguos, para denotar el número de mil ponían una $\mathrm{m}$ de las mayúsculas. (Pérez de Moya 1589, fol. 12r)

Haze también a mi propósito el gran estudio, vigilancia y trabajo que nuestros antepassados tuvieron por bien de tomar, sólo por venir en conoscimiento d'esta sciencia [matemática], y, en demás d'esto, la gran

10. "Durante el siglo XVI la tradición de origen clásico continuó siendo la base general del cultivo de las diferentes áreas científicas en la Europa occidental” (López Piñero 149) 
solicitud que para nuestro provecho tuvieron en dexárnosla por escrito, para que, no caresciendo de un tan alto bien, participássemos todos d'él. (Aurel, fol. IIr-v).

a) Pitágoras (Samos, 572 a. C.-Metaponto, 497 a. C.) y los pitagóricos

Conforme a la cuenta de los pitagóreos, las letras de el ABC tenían ciertos números, como paresce por Terenciano Mauro, y perdiose y quedaron solamente aquéllas que sirven de cuenta, que son éstas: I, V, X, L, C, D, con las quales, y las que de éstas se componen, se suele demostrar la summa que queremos d'esta manera: por I uno, por $\mathrm{V}$ cinco, por $\mathrm{X}$ diez, por $\mathrm{L}$ cinqüenta, por C ciento, por D quinientos. (Pérez de Moya 1562, 15)

Arithmética commúnmente se define que es una arte que trata de números y de sus passiones, por la qual arte procuravan alcançar aquellos philósophos pythagóricos todas las cosas que querían. Y a mi parescer no yvan muy engañados, según aquella sentencia (que dize) debaxo de tres cosas aver Dios dispuesto todo lo criado, conviene saber: número, peso y medida. (Pérez de Moya 1562, 687-88)

Por el número arithmético vino a alcançar Pythágoras los movimientos de los cielos y las concordancias y revoluciones que entre ellos avía. Cosa, cierto, que, aunque no uviera otro argumento sino éste, bastava para conoscer de quántos quilates sea esta arte [Aritmética] y quánto es lo que por ella se puede alcançar. Porque, dexando aparte el testimonio de tantos varones que la approvaron, como fue Pythágoras, Platón, Aristóteles, Sócrates y otros muchos, vemos ser tan necessaria a la vida humana que me atrevo a dezir ser una de las principales partes que se requieren para la conservación de la república. [...] Todo andaría tan confuso sin ella, que imagino que todas las cosas estarían en perpetua confusión. (Pérez de Moya 1562, 688)

b) Platón (Atenas, 427a. C.-347a. C.) ${ }^{11}$

11. "La importancia de Platón en la historia de la matemática deriva en gran parte de su papel como inspirador y director de otros matemáticos, pero quizá a él personalmente se deba la distinción neta y clara que hizo la antigua Grecia entre aritmética (en el sentido de teoría de números) y logística (o técnica de la computación). Platón consideraba a la logística como conveniente para el comerciante o para el hombre de guerra, que 'debe aprender el arte de los números o no sabrá cómo desplegar sus tropas'. El filósofo, en cambio, debe ser un aritmético 'porque tiene que conseguir salir del mar del cambio para establecer contacto con el verdadero ser”' (Boyer 123-24). 
Tan subtil, tan excelente

Es el arte del contar,

Tan dulce y tan eminente,

Tan fácil, lector prudente,

Qu'en las artes no hay su par.

Es tan alta, que Platón

En su puerta ansí escrivía:

Nadie oyga mi lición

Ni se tenga por varón,

$\underline{\text { Si contar ya no sabía. (Aurel, fol. Iv) }}$

Que la sciencia mathemática, entre todas las Artes liberales, muy Magnífico Señor, sea la más clara y la más cierta y necessaria es cosa evidente, pues sola ella (y principalmente lo que en ella se trata) es la que con más claras y abundosas demonstraciones se demuestra. Dexo aparte los freqüentíssimos testimonios para haver de confirmar mi opinión de aquel más que humano Platón, con los quales nos incita a querer y amar más a esta sciencia que a otra ninguna, pues nos muestra que, sin esta una, en ninguna manera los ánimos de los hombres pueden ser perfectamente incitados al conoscimiento de las cosas naturales, las quales quanto hagan al uso humano no ay quien no lo conozca. (Aurel, fol. IIr)

c) Aristóteles (Estagira, 384 a. C.-Calcis, 322 a. C.) ${ }^{12}$

Como dize Aristóteles, y número es un ayuntamiento o collectión de unidades, poderemos, por tanto, imaginar estos números y unidades en las líneas, y en las superficies y en los cuerpos por su división en partes. (Núñez, fol. 5v)

Y assí hallarás (si bien notares) no ser otra cosa el summar sino hazer de unidades diezes, y de diezes cientos, y de cientos millares, etc., la qual orden va d'esta suerte, que diez unos hazen un diez, diez diezes un

12. "Discípulo de Platón y preceptor de Alejandro Magno, es una figura destacada de la cultura griega de la segunda mitad del S. IV a. C. Su opinión sobre las matemáticas se distancia de la de Platón, pues según él no existen objetos matemáticos separados e independientes, sino que los conceptos de las matemáticas proceden de los cuerpos matemáticos por abstracción. Así, el matemático abstrae las propiedades físicas concretas de un objeto físico y de esta manera llega a las propiedades matemáticas que le compete estudiar" (Millán 44). 
ciento, diez cientos un millar, etc., como en la plática de los exemplos entenderás mejor. La causa por que todas las gentes cuentan por diezes Aristóteles la da por natural y necessaria. (Pérez de Moya 1562, 21)

Con todo, en la mención de autores clásicos, despunta la figura del matemático griego Euclides (330 a. C.-275 a. C.) y sus Elementos, obra que constituye "la base de la moderna civilización occidental" (Millán 21). Tal fue la influencia del heleno que en prácticamente todas las disciplinas y tratados científicotécnicos de la época renacentista se hace alusión a sus teorías.

Entre las citas que hacen referencia a Euclides es especialmente significativa en el campo de estudio que nos ocupa la que trata sobre la génesis de la aritmética, constituida por el concepto de "número", ${ }^{13}$ que el matemático define del siguiente modo:

Arithmética, la qual es sciencia de números [...]. Todo vocablo en Arithmética es subjeto y atribuido a número, cuya definición (según Euclides en el primero del séptimo) es una multitud compuesta de unidades, como 2, 3, 4, 5, etc., assí llamado porque, siendo, como es, uno solo indivisible, no tiene composición alguna ni es número, mas principio, reyna y fundamento de todo número. (Aurel, fol. 1r)

Hemos diffinido el Arithmética diziendo que es sciencia que trata de números; por tanto, conviene dezir qué cosa sea número y cómo se engendra. $Y$ assí digo que número (según Euclides) es una multitud compuesta de unidades, como 2, 3, 4, 5, 6, etc., y es a saber que, assí como del fluxo o movimiento del punto (según longitud) se describe y haze línea, assí de un allegamiento de unidades es hecho el número. (Pérez de Moya 1562, $3-4)$

Los tratadistas del Quinientos incluso explicitan la deuda que sus obras aritmético-algebraicas tienen con respecto a la terminología y las teorías plasmadas en los Elementos:

También es bien que sepas que he querido usar en muchas partes, mayormente en el capítulo $11^{\circ}$ y $22^{\circ}$, de los proprios términos de que usa

13. El concepto de "número" para los griegos se refería siempre a lo que hoy denominamos números naturales o enteros positivos. 
Euclides, y, assimesmo, nombrar las líneas por los nombres que él en su obra las nombra, pues veýa (aunque en la prática haze muy poco al caso) que en la theórica importava muy mucho a aquéllos que quisiessen aplicar sus ánimos a esta tan insigne sciencia, siguiendo las pisadas de Euclides. (Aurel, fol. IIIr)

Y porque estos fundamentos son de Euclides, que de todos los mathemáticos son recebidos, sacamos también d'aquý esta doctrina. (Núñez, fol. 66v)

$\mathrm{Y}$, en ocasiones, para una mejor comprensión de sus tratados, remiten a la lectura de las demostraciones euclídeas, puesto que en matemática conocer es demostrar y los Elementos "nos ofrecen el primer ejemplo en gran escala de ese fecundo juego de la razón, creador de nuevos conocimientos que se presentaban atraídos por la irresistible fuerza del raciocinio y cuya única finalidad es el conocimiento mismo" (Rey/Babini 76-77).

Al margen: La razón d'ésta se collige de la duodécima del séptimo de Euclides.

[...] Al margen: La razón d'esto de la 5 del 8 de Euclides se collige.

[...] Al margen: Lee la 17 del 8 de Euclides.

Al margen: Lee el 10 de Euclides. (Pérez de Moya 1562, 764)

\subsubsection{Modernos}

Por otro lado, destaca la intertextualidad e influencia de autores matemáticos italianos coetáneos ${ }^{14}$ para el avance y desarrollo del álgebra en la Península Ibérica del siglo XVI.

a) Luca Pacioli (Sansepolcro, 1445-Roma, 1517). Sin duda alguna, tanto la figura del fraile franciscano y matemático Luca Pacioli como su enciclopédica obra: Suma de arithmetica, geometria, proportioni et proportionalità (1494), la más destacada e influyente de las aritméticas publicadas en la Europa occidental (especialmente en lo que respecta a los algoritmos y notaciones algebraicas, ver Molina, 2016), son una de las referencias principales y constantes de los tratados matemáticos renacentistas. Núñez, por ejemplo, explicita en el pró-

14. Caunedo (59) evidencia en los tratados de aritmética práctica de la Europa occidental de la época estudiada "un notable grado de comunicación cultural entre las diversas comunidades científicas". 
logo y en las conclusiones de su obra la innegable herencia italiana presente en la misma (Molina 2015b), al mismo tiempo que sugiere ciertas correcciones, movidas por un afán pragmático y didáctico: "Fray Lucas trató este caso y resolviolo por modo muy cierto, pero erró por inadvertencia la cuenta de la primera parte y quarta, poniendo más en lugar de menos” (Núñez, fol. 215v). Del mismo modo, Aurel detalla los yerros cometidos por Pacioli e incluso, en ocasiones, amplía sus teorías:

Agora te quiero mostrar ocho reglas para las ocho ygualaciones, en las quales están fundadas las respuestas de nuestra Regla de la cosa o Arte mayor, dado que algunos ponen seis, como Fray Lucas del Burgo y otros diez, como Alberturcio de Saxonia. A mí, empero, me ha parecido tomar el medio arithmético entre 10 y 6 , que es 8 , pues por ellas entenderás las seis de Fray Lucas, y por las mesmas alcançarás las diez de Albertucio. Las quatro son simples, de dos quantidades; y las otras quatro compuestas, de tres quantidades, como aquí las verás de una en una. (Aurel, fol. 77v)

A pesar de los errores del franciscano y de que no llegara a resolver las ecuaciones cúbicas, Pacioli representa el paso del siglo XV al XVI, el gran siglo del Renacimiento y de los algebristas italianos: Gerolamo Cardano y Niccolò Fontana -más conocido por su sobrenombre, Tartaglia ${ }^{15}$-. Ellos hallaron la forma de resolver las ecuaciones de tercer y cuarto grado y rompieron, según Martín (84), "las amarras que hasta el momento retenían al álgebra y la consolidaron como una rama independiente de las matemáticas".

b) Gerolamo Cardano (Pavía, 1501-Roma, 1576). Este médico reputado, astrólogo acusado de herejía y profesor de matemáticas de varias universidades italianas, divulgó en el año 1545, con la publicación de su Ars Magna, la solución de la ecuación cúbica. Un avance "tan sorprendente e inesperado como este produjo un impacto tan fuerte en el mundo de los algebristas" (Boyer 362), que se suele considerar este año como el que marca el comienzo del periodo moderno en la matemática. No obstante, como veremos, él no fue su descubridor original.

15. En español "tartaja" o "tartamudo", defecto del habla producido por una perforación en la tráquea durante el saqueo de su ciudad natal, Brescia, por las tropas francesas de Gastón de Fix (ver Martín 149). 
c) Niccolò Fontana o Tartaglia (Brescia, 1499-Venecia, 1557). Este autodidacta matemático italiano, conocido por el apodo que deriva de su defecto físico del habla, Tartaglia (es decir, 'tartaja'), llegó a ser uno de los científicos más sobresalientes del contexto europeo del XVI. Enseñó y divulgó matemáticas en varias ciudades italianas, entre las que destacan Verona, Venecia y Brescia (su ciudad natal) y alcanzó notable éxito y fama por ser el creador de un método capaz de satisfacer la solución de la ecuación cúbica, que llegó a los oídos de Cardano. Estos dos matemáticos italianos pasaron a la historia por ser los protagonistas de la divulgación de un descubrimiento que hasta el momento se había considerado imposible: la resolución de la ecuación cúbica, ${ }^{16}$ esto es, de el cubo y la cosa igual a número. ${ }^{17}$

Este gran avance matemático, sin embargo, no estuvo exento de polémica, suscitada por las disputas entre Tartaglia, descubridor de las operaciones necesarias para la resolución de este tipo de ecuaciones, y Cardano, quien las publicó en su Ars Magna y se atribuyó los méritos de tal logro tras convencer a Tartaglia para que le confiara sus investigaciones. ${ }^{18}$ Tan sonados fueron los enfrentamientos entre ambos autores y el aventajado alumno del segundo, Ludovico Ferrari, ${ }^{19}$ que nuestros textos dan cuenta de los entresijos de esta historia, narrada por Núñez en su Álgebra del siguiente modo:

Nicolao Tartalla fue grandíssimo arithmético, y halló capítulos nuevos de cosas y cubo yguales a número, y los compañeros d'éste, de los quales haze mención en el su libro De las invenciones, los quales capítulos no son aún divulgados; y de creer es que, si biviendo él, divulgara esta su Álgebra que anda impresa, la embiara tan emendada que no uviera en ella qué apuntar [...]. Dize en el dicho libro De las invenciones que, aviéndole pedido Hierónymo Cardano regla para saber el valor de la cosa, quando cosas y cubo son yguales a número, le mostrara este su invento en un soneto [...].Y dize más, que la declaración d'esta regla le

16. En realidad, su primer descubridor fue Scipione del Ferro, ¿'1505-1515? (ver Martín 85-108).

17. Que en notación simbólica actual se representa: $x^{3}+p x=q$.

18. Como detalla Núñez (fol. 331v), cifradas en el siguiente soneto: "Quando chel cubo con le cose apresso / Se agualia a qualche numero discreto / Trovan dui altri differenti in esso, / Da poi terra i questo per consueto / Ch'el lor producto sempre si eguale / $\mathrm{Al}$ terzo cubo delle cose neto, / El residuo poi suo generale / Delli lor lati cubi ben sotrati / Varra la sua cosa principale".

19. El cual se enfrentó a Tartaglia mediante varios carteles y, finalmente, en un duelo público, donde demostró tener un nivel intelectual muy superior a su contricante. De tal manera que será, junto a Rafael Bombelli, el protagonista de la resolución de las ecuaciones de cuarto grado. 
dio en este exemplo 1 cubo más 3 cosas son yguales a 10 , y que le dio el Cardano su fe y prometió, jurándole por los sanctos Evangelios, que no divulgaría esta su regla en ninguna obra que publicasse, puesto que en ella quisiese confessar que d'él la deprendiera. $\underline{Y}$ porque después d'esto el dicho Hierónymo Cardano divulgó una obra en la qual trae la misma regla, confessando que la deprendiera del mismo Nicolao Tartalla, pero que teniendo la regla fácilmente por sí hallara la demonstración, enojose mucho d'esto, o porque que no le cumplió la promessa. (fols. 331r-32v)

En estas líneas se advierte que el matemático portugués conocía perfectamente los hechos, pero también las obras y el estilo de estos autores, hasta el punto de ofrecer una lúcida crítica:

Vino otra Suma de Hierónymo Cardano, la qual compuso con emulación de Fray Lucas, porque haze un capítulo de sus yerros, en tanto que le nota por yerros los descuidos o inadvertencias en el summar, partir o multiplicar, en que no cabía ignorancia. Este autor tuvo en el principio orden, mas después escribe confusamente, y haze de todo una ensalada mal hecha, y después embió otro libro de Álgebra, que es un chaos.

Después d'éstos, Nicolao Tartalla, muy gran maestro de cuenta y buen geómetra, notó los yerros de entrambos en los libros que compuso y, después de su muerte, vino al presente un libro, que en su casa se halló, en el qual separadamente trata de Álgebra. El qual libro, en la orden y clareza y en el estylo, muestra ser suyo, principalmente que va en él allegando lo que en los otros libros avía escripto, y por él se puede muy mejor deprender esta arte que por los libros de Fray Lucas y Cardano. (fol. 322r)

$\mathrm{Al}$ igual que Pacioli, ninguno de los autores matemáticos hispanos del Quinientos fue capaz de dar solución a la ecuación cúbica. Entre otros, Núñez, por ejemplo, consciente de su carencia, da fin a su tratado sobre álgebra justificándose y disculpándose por tal falta y postulando la posibilidad de mostrarla en alguna de sus futuras obras:

Y aquý acabo esta obra, supplicando a los lectores que no me quieran dar culpa por no traer esta regla de cosa y cubo yguales a número, y las otras de dignidades disproporcionales, por sus principios, porque el trabajo era 
grande y muy chico el loor, principalmente no me contendando aquella manera de notificar el valor de la cosa. Allá lo hallarán todo tratado por el Cardano, o bien o mal. Y, si Dios nos diere a entender otro mejor modo, traerlo emos en otro libro. (fol. 339r)

Finalmente, terminamos este análisis con las recomendaciones de Juan de Belveder, quien, en su peculiar aritmética comercial en torno a las reducciones de plata y oro, cita, a finales de la centuria, a los matemáticos estudiados entre las autoridades de este campo del saber que hoy nos ocupa:

Porque no se me atribuya a descuydo, negligencia o a otro fin, y porque no fuesse tan sola esta obra de las reduciones de monedas [...], me obligo a poner aquí algunas reglas de abreviaturas ordinarias y necessarias para las contrataciones d'estos reynos, con otras breves del Arte Menor y Mayor, de argumentos breves y fáciles, para que con ellos en halgo se puedan entretener los curiosos contadores, que por no hazer volumen de escriptura he dexado de poner algunas reglas de mucho estudio y consideración, remitiendo en lo demás a los que d'este harte fueren afficionados, que lean las Matemáticas de Moya del Arte Mayor y Menor, como las de Tartalla, el alemán [Aurel], ${ }^{20}$ Euclides, Oroncio, Burgo y Ortega. (fol. 190v)

\section{CONCLUSIÓN}

Como anticipábamos, el desarrollo de las ciencias exactas en el Quinientos hispano estuvo marcado fundamentalmente por las aplicaciones prácticas del saber matemático a las demandas sociales; una de las que mayor relevancia tuvo en la España renacentista fue el cálculo mercantil, de ahí la proliferación de libros publicados sobre contabilidad o aritméticas prácticas, entre las que se integra el corpus textual analizado a lo largo de estas páginas.

El objetivo y el gran mérito de estos tratados aritmético-algebraicos fue la democratización del cálculo y la generalización del uso de los numerales indo-arábigos, así como del principio posicional de base decimal y los sofisticados algoritmos de origen oriental. Este sistema, mucho más eficaz y aventajado que el tradicional uso del ábaco, la mano o los numerales romanos,

20. El corchete es nuestro. 
permitió que el secretismo medieval en torno a las cuentas se propagara, en el Siglo de Oro, a una franja más extensa de la población.

Uno de los hechos que contribuyó de manera trascendental a la aritmetización de la sociedad quinientista fue el empleo de la lengua vulgar como vehículo de expresión científica, con el fin de poner la ciencia al alcance de todo el mundo. Efectivamente, como hemos podido comprobar, las cinco obras revisadas son textos didácticos, marcados por un estilo claro y sencillo, en el que abundan, entre otras, las explicaciones, comparaciones, ejemplos, instrucciones, demostraciones y definiciones de los objetos, ideas y conceptos matemáticos que, por primera vez, se designan y/o describen en español. Este conjunto textual presenta, por tanto, un denorminador común: la llaneza de su retórica -característica ya proclamada por algunos humanistas coetáneos, como Juan de Valdés, que, en el Diálogo de la lengua (223), defiende el uso de un "estilo natural, y sin afectación ninguna"-, ${ }^{21}$ pues, como reconoce Núñez, "no es nuestra intención escrevir para los doctos, los quales de nuestra escriptura no ternán necessidad" (fol. 46r).

En suma, la preocupación por el rigor, la precisión y la transparencia del sentido de los conceptos relativos a la aritmética y el álgebra que introducen, así como por los recursos básicos de la retórica, entre los que se cuenta, lógicamente, la claridad de la argumentación (Alburquerque 65), aunados a la finalidad eminentemente pragmática y el espíritu didáctico de Ortega, Aurel, Pérez de Moya y Núñez Salaciense son rasgos definitorios de un discurso, el matemático, y de una época, la renacentista, fundamentales tanto para la dignificación e intelectualización de las lenguas vulgares como para el asentamiento, desarrollo y difusión de saberes tradicionalmente transmitidos en latín a una minoritaria élite culta.

\section{OBRAS CITADAS}

\section{FUENTES}

Aurel, Marco. Libro primero de Arithmética algebrática. Valencia: Joán de Mey, 1552.

Belveder, Joan de. Libro general de la reduciones de plata y oro. Lima: Antonio Ricardo, 1597.

21. Quien apunta, además: "solamente tengo cuidado de usar de vocablos que signifiquen bien lo que quiero decir, y dígolo quanto más llanamente me es posible" (223). 
Núñez, Pedro. Libro de Algebra en Arithmética y Geometría. Anvers: Herederos de Arnoldo Birckman, 1567.

Ortega, Juan. Conpusición de la arte de la Arismética y de Geometría. Lyon: Maistro Nicolau de Benedictis (por Joannes Trinxer), 1512.

Pérez de Moya, Juan. Arithmética práctica y speculativa. Salamanca: Mathías Gast, 1562.

Pérez de Moya, Juan. Manual de contadores. Madrid: Pedro Madrigal, 1589.

\section{ESTUDIOS}

Alburquerque, Luis. El arte de hablar en público: seis retóricas famosas del siglo XVI: Nebrija, Salinas, G. Matamoros, Suárez, Segura y Guzmán. Madrid: Visor, 1995.

Boyer, Carl B. Historia de la matemática. 2. ${ }^{\text {a }}$ ed. Madrid: Alianza, 2003.

Caunedo, Betsabé. "El arte del alguarismo en la Europa medieval". El arte del alguarismo: un libro castellano de aritmética comercial y de ensayo de la moneda del siglo XIV (Ms. 46 de la Real colegiata de San Isidoro de León). Eds. Betsabé Caunedo y Rafael Córdoba. Salamanca: Junta de Castilla y León, 2000. 23-82.

DICTER: Mancho Duque, M. Jesús, dir. Diccionario de la ciencia y de la técnica del Renacimiento. Salamanca: Ediciones Universidad de Salamanca, 20002013. 15 de mayo de 2015. <http://dicter.usal.es/>.

Docampo, Javier. "La formación matemática del mercader catalán 1380-1521: análisis de fuentes manuscritas". Tesis doctoral inédita. Universidade de Santiago de Compostela, 2004.

Etayo Miqueo, José Javier. "El lenguaje de las matemáticas". Aproximaciones al lenguaje de la ciencia. Ed. Bertha M. Gutiérrez Rodilla. Burgos: Colección Beltenebros, 2003. 345-370.

Flórez, Cirilo. "Ciencias, siglos XV-XVII". Historia de la Universidad de Salamanca, III: Saberes y confluencias. Ed. Luis Enrique Rodríguez-San Pedro Bezares. Salamanca: Ediciones Universidad de Salamanca, 2006, 409-33.

Kristeller, Paul Oscar. El pensamiento renacentista y sus fuentes. México: Fondo de Cultura Económica, 1982.

Lausberg, Heinrich. Manual de retórica literaria. Madrid: Gredos, 1995.

Leitão, Henrique. "Pedro Nunes e o Libro de Álgebra". Quaderns d'bistòria de l'enginyeria 10 (2010): 9-19.

López Piñero, José M. ${ }^{a}$. Ciencia y técnica en la sociedad española de los siglos XVI y XVII. Barcelona: Labor, 1979. 
Maravall, José Antonio. Estado moderno y mentalidad social. Madrid: Revista de Occidente, 1972.

Martín Casalderrey, Francisco. Cardano y Tartaglia: las matemáticas en el Renacimiento italiano. Madrid: Nivola, 2000.

Millán Gasca, Ana. Euclides: la fuerza del razonamiento matemático. Madrid: Nivola, 2007.

Molina Sangüesa, Itziar. "Las matemáticas en el Renacimiento hispano: estudio léxico y glosario". Tesis doctoral inédita. Universidad de Salamanca, $2015 \mathrm{a}$.

Molina Sangüesa, Itziar. "Tradición e innovación en el ámbito de la divulgación matemática del Quinientos". La ciencia como diálogo entre teorías, textos y lenguas. Eds. Jenny Brumme y Carmen López Ferrero. Berlín: Frank \& Timme, 2015b. 31-48.

Molina Sangüesa, Itziar. Letras, números e incógnitas: estudio de las voces aritmético-algebraicas del Renacimiento. Madrid: Iberoamericana/ Frankfurt am Main: Vervuert, 2017.

Molina Sangüesa, Itziar. "La designación de las potencias de la incógnita: algunas cuestiones sobre el tránsito del álgebra retórica al álgebra sincopada en el Renacimiento hispano". Arbor: ciencia, pensamiento, cultura 192/777 (2016): a293.

Picatoste y Rodríguez, Felipe. Apuntes para una biblioteca científica española del siglo XVI. Madrid: Imprenta y Fundación Manuel Tello, 1891.

Rey Pastor, Julio. Los matemáticos españoles del siglo XVI. Madrid: Biblioteca Scientia, 1926.

Rey Pastor, Julio, y José Babini. Historia de la matemática: de la antigüedad a la baja Edad Media. Barcelona: Gedisa, 2000.

Rodríguez Vidal, Rafael. Diálogos de Aritmética práctica y especulativa (1562), Bachiller fuan Pérez de Moya. Zaragoza: Prensas Universitarias de Zaragoza, 1987.

Salavert Fabiani, Vicente. "Introducción a la historia de la aritmética práctica en la Corona de Aragón del siglo XVI". Dynamis: Acta Hispanica ad Medicinae Scientiarumque Historiam Illustrandam 10 (1990): 63-91.

Valdés, Juan de. Diálogo de la lengua. Ed. Cristina Barbolani. 4. ${ }^{a}$ ed. Madrid: Cátedra, 1990.

Valladares, Aurelio. "El Bachiller Juan Pérez de Moya: apuntes bio-bibliográficos”. Boletín del Instituto de Estudios Giennenses 165 (1997): 371-412. 